Georgian Mathematical Journal

Volume 8 (2001), Number 4, 669-681

\title{
ON THE EXISTENCE OF SINGULAR SOLUTIONS
}

\author{
M. BARTUŠEK AND J. OSIČKA
}

\begin{abstract}
Sufficient conditions are given, under which the equation $y^{(n)}=$ $f\left(t, y, y^{\prime}, \ldots, y^{(l)}\right) g\left(y^{(n-1)}\right)$ has a singular solution $y[T, \tau) \rightarrow \mathbf{R}, \tau<\infty$ satisfying $\lim _{t \rightarrow \tau_{-}} y^{(i)}(t)=c_{i} \in \mathbf{R}, i=0,1, \ldots, l$ and $\lim _{t \rightarrow \tau_{-}}\left|y^{(j)}(t)\right|=\infty$ for $j=l+1, \ldots, n-1$ where $l \in\{0,1, \ldots, n-2\}$.
\end{abstract}

2000 Mathematics Subject Classification: 34C11.

Key words and phrases: Singular solution, fourth order differential equation, black hole solution.

\section{INTRODUCTION}

Consider the $n$-th order differential equation

$$
y^{(n)}=f\left(t, y, y^{\prime}, \ldots, y^{(l)}\right) g\left(y^{(n-1)}\right),
$$

where $n \geq 2, l \in\{0,1, \ldots, n-2\}, f \in C^{0}\left(\mathbf{R}_{+} \times \mathbf{R}^{l+1}\right), g \in C^{0}(\mathbf{R}), \mathbf{R}_{+}=[0, \infty)$, $\mathbf{R}=(-\infty, \infty)$ and there exists $\alpha \in\{-1,1\}$ such that

$$
\alpha f\left(t, x_{1}, \ldots, x_{l+1}\right) x_{1}>0 \quad \text { for } \quad x_{1} \neq 0 .
$$

A solution $y$ defined on the interval $[T, \tau) \subset \mathbf{R}_{+}$is called singular if $\tau<\infty$ and $y$ cannot be defined for $t=\tau$.

The problem of the existence of singular solutions satisfying the Cauchy initial-value problem and their asymptotic behaviour is thoroughly studied in [4] for the second order Emden-Fowler equation

$$
y^{\prime \prime}=r(t)|y|^{\lambda} \operatorname{sgn} y, \quad r(t) \geq 0 .
$$

In the common case for (1), the profound investigations are carried out in [5]. All these results concern the case $\alpha=1$. For $\alpha=-1$, sufficient conditions are given in [2], under which singular solutions of

$$
y^{(n)}=r(t)|y|^{\lambda} \operatorname{sgn} y, \quad n \geq 2, \quad r \leq 0,
$$

exist.

Another problem concerning singular solutions is solved in [3] $(n=2$, $\left.f\left(t, x_{1}, \ldots, x_{l}\right) \equiv r(t)\left|x_{1}\right|^{\sigma} \operatorname{sgn} x_{1}, g(x)=|x|^{\lambda}\right)$ and in [1] in the case $l=n-2$. 
Let $\tau \in(0, \infty)$. Sufficient and/or necessary conditions are given there, under which a singular solution $y$ exists with given asymptotic behaviour at the left-hand side point $\tau$ of the definition interval $c_{i} \in \mathbf{R}$,

$$
\lim _{t \rightarrow \tau_{-}} y^{(i)}(t)=c_{i} \quad \text { for } \quad i=0,1, \ldots, n-2, \quad \lim _{t \rightarrow \tau_{-}}\left|y^{(n-1)}(t)\right|=\infty .
$$

In the present paper this result is generalized to the case in which we seek a singular solution $y$ satisfying the condition

$$
\begin{gathered}
\tau \in(0, \infty), \quad c_{i} \in \mathbf{R} ; \quad \lim _{t \rightarrow \tau_{-}} y^{(i)}(t)=c_{i}, \quad i=0,1, \ldots, l \\
\lim _{t \rightarrow \tau_{-}}\left|y^{(j)}(t)\right|=\infty \quad \text { for } \quad j=l+1, \ldots, n-1 .
\end{gathered}
$$

Note that in [3] such solutions are called blackhole solutions (for $n=2$ and $l=0)$.

Denote by $[[a]]$ the entire part of the number $a$.

\section{Main Results}

Let $y$ be a solution of (1) satisfying (5). Since, according to (2) $f\left(t, c_{0}, c_{1}, \ldots, c_{l}\right) \neq 0$ if and only if $c_{0} \neq 0$, we ought to divide our investigation into two cases $c_{0} \neq 0$ and $c_{0}=0$ for which the results are different.

Let $c_{0} \neq 0$. The following theorem gives a necessary condition for the existence of a solution of (1), (5).

Theorem 1. Let $c_{0} \neq 0, M \in(0, \infty), K \in(0,1], \lambda \leq 2$ for $l=n-2$,

$$
\begin{gathered}
\lambda \bar{\epsilon}\left(1+\frac{1}{n-l-1}, 1+\frac{1}{n-l-2}\right] \quad \text { for } \quad<n-2, \\
K|x|^{\lambda} \leq g(x) \leq|x|^{\lambda} \quad \text { for } \quad|x| \geq M .
\end{gathered}
$$

Then equation (1) has no singular solution y satisfying (5).

The next theorem shows that in the opposite case in (6) problem (1), (5) is solvable.

Theorem 2. Let $\tau \in(0, \infty), c_{0} \neq 0, M \in(0, \infty), \beta=\alpha \operatorname{sgn} c_{0}, \lambda>2$ for $l=n-2$,

$$
1+\frac{1}{n-l-1}<\lambda \leq 1+\frac{1}{n-l-2} \quad \text { for } \quad l<n-2
$$

and

$$
g(x) \geq|x|^{\lambda} \quad \text { for } \quad \beta x \geq M .
$$

Then there exists a singular solution y of (1) satisfying (5) which is defined in a left neighborhood of $\tau$.

If, moreover, $\varepsilon>0, g(x)>0$ for $\beta x \in(0, \varepsilon]$,

$$
l+\frac{1-\alpha}{2} \quad \text { is odd, } \quad(-1)^{i} c_{i} c_{0} \geq 0 \quad \text { for } \quad i=1,2, \ldots, l
$$


and

$$
\left|\int_{0}^{\beta \varepsilon} \frac{d s}{g(s)}\right|=\infty,
$$

then $y$ is defined on the interval $[0, \tau)$.

Corollary 1. Let $c_{0} \neq 0, M \in(0, \infty)$ and

$$
g(x)=|x|^{\lambda} \quad \text { for } \quad|x| \geq M .
$$

Then (1) has a singular solution y satisfying (5) if and only if (7) is valid.

Corollary 2. Let $\lambda>1+\frac{1}{n-1}$ and $M \in \mathbf{R}_{+}$be such that

$$
g(x) \geq x^{\lambda} \quad \text { for } \quad x \geq M .
$$

Then (1) has a singular solution.

Remark. For $\alpha=1$ the conclusion of Corollary 2 is known, see, e.g., [6, Theorem 11.3]. For $\alpha=-1$ it generalizes Corollary 1 in [1].

The following two theorems solve the same problem in the case

$$
\beta \in\{-1,1\}, \quad c_{0}=0, \quad(-1)^{i} \beta c_{i} \geq 0 \quad \text { for } \quad i=1,2, \ldots, l .
$$

Theorem 3. Let $\tau \in(0, \infty), \sigma>0, \varepsilon>0, M \in(0, \infty), \bar{M} \in(0, \infty)$,

$$
\begin{gathered}
l-\frac{1-\alpha}{2} \quad \text { be odd } \\
2+(n-2) \sigma<\lambda \quad \text { for } l=n-2, \\
1+\frac{l \sigma+1}{n-l-1}<\lambda \leq 1+\frac{(l+1) \sigma+1}{n-l-2} \quad \text { for } l<n-2,
\end{gathered}
$$

(8) and (11) hold. Further, let

$$
\left|f\left(t, x_{1}, \ldots, x_{l+1}\right)\right| \geq \bar{M}\left|x_{1}\right|^{\sigma}
$$

for $t \in[0, \tau], \beta x_{1} \in[0, \varepsilon],(-1)^{j} \beta x_{j+1} \in\left[(-1)^{j} \beta c_{j},(-1)^{j} \beta c_{j}+\varepsilon\right], j=1, \ldots, l$.

Then there exists a singular solution y of (1) satisfying (5), which is defined in a left neighborhood of $\tau$.

If, moreover, $g(x)>0$ for $\beta x \in(0, \varepsilon]$ and $(10)$ holds, then $y$ is defined on the interval $[0, \tau)$.

Theorem 4. Let $\sigma>0, c_{i}=0$ for $i=0,1, \ldots, l, M \in(0, \infty), \varepsilon>0$, $\alpha \in\{-1,1\}, r \in C^{0}\left(\mathbf{R}_{+}\right), \alpha r(t)>0$ on $\mathbf{R}_{+}$, (12) hold and

$$
g(x)=|x|^{\lambda} \quad \text { for } \quad|x| \geq M \text {. }
$$

Then the equation

$$
y^{(n)}=r(t)|y|^{\sigma} g\left(y^{(n-1)}\right) \operatorname{sgn} y
$$

has a solution y satisfying (1), (5) if and only if (13) is valid. 
The following proposition shows that assumption (12) in Theorems 3 and 4 is important.

Proposition. Let $c_{i}=0, i=0,1, \ldots, l$ and $l-\frac{\alpha-1}{2}$ be even. Let $g(x) \geq 0$ on $\mathbf{R}$. Then equation (1) has no solution satisfying (1), (5).

In this paper the main assumptions are imposed on the function $g$ depending on $y^{(n-1)}$. But solutions of (1), (5) may exist for the equation

$$
y^{(n)}=f\left(t, y, \ldots, y^{(j)}\right), \quad j \in\{l+1, \ldots, n-1\},
$$

too. From this we formulate an open problem.

Open problem. To study the existence of a solution satisfying (1), (5) of equation (16).

\section{Lemmas AND PROOFS}

We need the next two lemmas.

Lemma 1. Let $[a, b] \subset \mathbf{R}_{+}, \phi \in C^{0}[a, b]$ and $\tilde{f} \in C^{0}\left([a, b] \times \mathbf{R}^{n}\right)$ be such that

$$
\tilde{f}\left(t, x_{1}, \ldots, x_{n}\right) \mid \leq \phi(t), \quad t \in[a, b], \quad x_{i} \in \mathbf{R}, \quad i=1, \ldots, n .
$$

Then for arbitrary $\gamma_{i} \in \mathbf{R}, i=0,1, \ldots, n-1$, the equation

$$
u^{(n)}=\tilde{f}\left(t, u, u^{\prime}, \ldots, u^{(n-1)}\right)
$$

has at least one solution satisfying the boundary value conditions

$$
\begin{aligned}
& u^{(i)}(b)=\gamma_{i} \quad \text { for } \quad i=0,1, \ldots, l+1 \\
& u^{(j)}(a)=\gamma_{j+1} \quad \text { for } \quad j=l+1, \ldots, n-2 .
\end{aligned}
$$

Proof. It follows, e.g., from [6, Lemma 10.1] since the homogeneous problem $u^{(n)}=0, \quad u^{(i)}(b)=u^{(j)}(a)=0 \quad$ for $\quad i=0,1, \ldots, l+1 ; \quad j=l+1, \ldots, n-2$, has a trivial solution only.

The following Kolmogorov-Horny type inequality is a very useful tool (see, e.g., the proof of Lemma 5.2 in [6]).

Lemma 2. Let $[a, b] \subset \mathbf{R}_{+}, a<b, m \geq 2$ be an integer, $u \in C^{m}[a, b]$, and let $u^{(j)}$ have zero in the interval $[a, b]$ for $j=1, \ldots, m-1$. Then

$$
\rho_{i} \leq 2^{i(m-i)} \rho_{0}^{\frac{m-i}{m}} \rho_{m}^{\frac{i}{m}}, \quad i=1,2, \ldots, m-1
$$

where

$$
\rho_{i}=\max \left\{\left|u^{(i)}(t)\right| a \leq t \leq b\right\}, \quad i=0,1, \ldots, m .
$$


Proof of Theorem 1. Let for simplicity $c_{0}>0$ and $\alpha=1$. Put $\lambda_{1}=\frac{1}{\lambda-1}$ and let $y\left[\tau_{1}, \tau\right) \rightarrow \mathbf{R}$ be a solution of (1), (5). Then, according to (1) and (2) $\lim _{t \rightarrow \tau_{-}} y^{(j)}(t)=\infty$ for $j=l+1, \ldots, n$. Let $T \in\left[\tau_{1}, \tau\right)$ be such that

$$
\begin{gathered}
y(t) \geq \frac{c_{0}}{2} \text { on }[T, \tau), \quad y^{(j)}(T) \geq 0, \quad j=l+1, l+2, \ldots, n-2, \\
y^{(n-1)}(T) \geq M .
\end{gathered}
$$

By this and the boundedness of $y^{(i)}(t), i=0,1, \ldots, l$, we obtain from (1)

$$
y^{(n)}(t) \leq M_{1}\left[y^{(n-1)}(t)\right]^{\lambda}, \quad t \in[T, \tau),
$$

where $M_{1}$ is a suitable constant. Let $\lambda \leq 1+\frac{1}{n-l-1}$. Hence the integration on the interval $[t, \tau)$ yields

$$
y^{(n-1)}(t) \geq\left[(\lambda-1) M_{1}(\tau-t)\right]^{-\lambda_{1}}, \quad t \in[T, \tau) .
$$

Hence $n-l-1 \leq \lambda_{1}$, and the Taylor Series Theorem, (17) and (18) yield

$$
\begin{aligned}
c_{l} & =y^{(l)}(\tau)=\sum_{i=0}^{n-l-2} \frac{y^{(l+i)}(T)}{i !}(\tau-T)^{i}+\int_{T}^{\tau} \frac{(\tau-s)^{n-l-2}}{(n-l-2) !} y^{(n-1)}(s) d s \\
& \geq y^{(l)}(T)+M_{2} \int_{T}^{\tau}(\tau-s)^{n-l-2-\lambda_{1}} d s=\infty,
\end{aligned}
$$

where

$$
M_{2}=\frac{\left[(\lambda-1) M_{1}\right]^{-\lambda_{1}}}{(n-l-2) !} .
$$

Hence a solution $y$ satisfying (1), (5) does not exist in this case.

Let $l<n-2$ and $\lambda>1+\frac{1}{n-l-2}$. Hence $n-l-2-\lambda_{1}>0$. Then, similarly to (18), we can prove that

$$
y^{(n-1)}(t) \leq\left[(\lambda-1) M_{3}(\tau-t)\right]^{-\lambda_{1}}, \quad t \in[T, \tau),
$$

where $M_{3}=\min \left\{K f\left(t, y(t), \ldots, y^{(l)}(t)\right) T \leq t \leq \tau\right\}>0$.

From this the Taylor Series Theorem yields

$$
\begin{aligned}
\infty & =y^{(l+1)}(\tau)=\sum_{i=0}^{n-l-3} \frac{y^{(l+i+1)}}{i !}(\tau-T)^{i}+\int_{T}^{\tau} \frac{(\tau-s)^{n-l-3}}{(n-l-3) !} y^{(n-1)}(s) d s \\
& \leq M_{4}+M_{5} \int_{T}^{\tau}(\tau-s)^{n-l-3-\lambda_{1}} d s<\infty
\end{aligned}
$$

as $n-l-3-\lambda_{1}>-1 ; M_{4}$ and $M_{5}$ are positive constants. The contradiction obtained proves that a singular solution does not exist.

Proof of Theorem 2. For $l=n-2$ we proved the statement in [1]. Thus let $l<n-2$ and, first, we prove the result for (7) with $\lambda \neq 1+\frac{1}{n-l-2}$. 
We prove the statement for $\alpha=1$ and $c_{0}>0$; thus $\beta=1$. For the other cases the proof is similar.

Let

$$
N>2 \max \left(c_{0},\left|c_{1}\right|, \ldots,\left|c_{l}\right|\right), \quad k_{0}>[[2 M]],
$$

$D=\left\{\left[x_{1}, \ldots, x_{l+1}\right] \frac{c_{0}}{2} \leq x_{1} \leq c_{0},\left|x_{j}\right| \leq N \quad\right.$ for $\left.\quad j=2, \ldots, l+1\right\}$,

$M_{1}=\min \left\{f\left(t, x_{1}, \ldots, x_{l+1}\right) t \in[0, \tau],\left[x_{1}, \ldots, x_{l+1}\right] \in D\right\}>0$,

$M_{2}=\max \left\{f\left(t, x_{1}, \ldots, x_{l+1}\right) t \in[0, \tau],\left[x_{1}, \ldots, x_{l+1}\right] \in D\right\}$,

$M_{3}=2\left[(\lambda-1) M_{1}\right]^{-\lambda_{1}}, \lambda_{1}=\frac{1}{\lambda-1}, \bar{\lambda}=n-l-1-\lambda_{1}>0$,

$N_{1}=2^{n-l-2} M_{3}^{\frac{1}{n-l-1}}\left[1-\frac{\lambda_{1}}{n-l-1}\right]^{-1}$.

Further, let $T \in[0, \tau)$ be such that

$$
\begin{gathered}
\tau-T<\left(\frac{M_{3}}{M}\right)^{\lambda-1}, \quad(\tau-T)^{n-l-2}<\frac{k_{0}}{M}, \\
\tau-T<\frac{1}{M_{2}} \int_{M}^{2 M} \frac{d s}{g(s)}, \\
(\tau-T)^{\bar{\lambda}} \leq\left(2 N_{1}\right)^{-n+l+1} N, \\
\sum_{r=i+1}^{l-1}\left|c_{r}\right| \frac{(\tau-T)^{r-i}}{(r-i) !}+N \frac{(\tau-T)^{l-i}}{(l-i) !} \leq \frac{N}{2}, \quad i=0,1, \ldots, l-1, \\
\sum_{r=1}^{l-1}\left|c_{r}\right| \frac{(\tau-T)^{r}}{r !}+N \frac{(\tau-T)^{l}}{l !} \leq \frac{c_{0}}{2} .
\end{gathered}
$$

Denote $J=[T, \tau)$ and note that due to $\bar{\lambda}>0, T$ exists.

Consider the auxilliary two-point boundary-value problem $k \in\left\{k_{0}, k_{0}+\right.$ $1, \ldots\}$,

$$
\begin{gathered}
y^{(n)}=f\left(t, \Phi_{0}(y), \Phi_{1}\left(y^{\prime}\right), \ldots, \Phi_{1}\left(y^{(l)}\right)\right) g\left(\Phi_{2}\left(t, y^{(n-1)}\right)\right), \\
y^{(i)}(\tau)=c_{i}, \quad i=0,1, \ldots, l ; \quad y^{(l+1)}(\tau)=k ; \\
y^{(j)}(T)=0, \quad j=l+1, \ldots, n-2, \quad t \in J,
\end{gathered}
$$

where

$$
\begin{aligned}
& \Phi_{0}(s)= \begin{cases}s & \text { for } \frac{c_{0}}{2} \leq s \leq N, \\
N & \text { for } s>N, \\
\frac{c_{0}}{2} & \text { for } s<\frac{c_{0}}{2},\end{cases} \\
& \Phi_{1}(s)= \begin{cases}s & \text { for }|s| \leq N, \\
N \operatorname{sgn} s & \text { for }|s|>N\end{cases}
\end{aligned}
$$


and

$$
\Phi_{2}(t, s)= \begin{cases}s & \text { for } M \leq s \leq M_{3}(\tau-t)^{-\lambda_{1}} \\ M_{3}(\tau-t)^{-\frac{1}{\lambda-1}} & \text { for } s>M_{3}(\tau-t)^{-\lambda_{1}} \\ M & \text { for } s<M\end{cases}
$$

Note that due to (21) $\Phi_{2}$ is well defined.

To prove the existence of a solution of (26), let us consider the sequence of boundary value problems

$$
\begin{gathered}
\bar{m}_{0}>\frac{1}{\tau-t}, \quad m \in\left\{\bar{m}_{0}, \bar{m}_{0}+1, \ldots\right\}, \quad \tau_{m}=\tau-\frac{1}{m}, \\
z^{(n)}=F\left(t, z, z^{\prime}, \ldots, z^{(l)}, z^{(n-1)}\right), \\
z^{(i)}\left(\tau_{m}\right)=c_{i}, \quad i=0,1, \ldots, l, \quad z^{(l+1)}\left(\tau_{m}\right)=k, \\
z^{(j)}(T)=0, \quad j=l+1, \ldots, n-2,
\end{gathered}
$$

where

$$
F\left(t, x_{1}, \ldots, x_{l+2}\right)=f\left(t, \Phi_{0}\left(x_{1}\right), \Phi_{1}\left(x_{2}\right), \ldots, \Phi_{1}\left(x_{l+1}\right)\right) g\left(\Phi_{2}\left(t, x_{l+2}\right)\right) .
$$

Since

$$
\left|F\left(t, x_{1}, \ldots, x_{l+2}\right)\right| \leq M_{2} \max _{T \leq \bar{t} \leq t} \max _{M \leq s \leq M_{3}(\tau-\bar{t})^{-\lambda_{1}}} g(\bar{t}, s), \quad t \in\left[T, \tau_{m}\right],
$$

(30) has a solution $z_{m}$ according to Lemma 1.

Further, we estimate $z_{m}^{(n-1)}$. Let $J_{m}=\left[T, \tau_{m}\right]$. First we prove that

$$
z_{m}^{(n-1)}(t)<M_{3}(\tau-t)^{-\lambda_{1}}, \quad t \in\left[T, \tau_{m}\right),
$$

for large $m$, say $m \geq \bar{m}_{0}$. If (31) is not valid, then either

(i) there exists $t_{1} \in\left[T, \tau_{m}\right)$ such that

$$
z_{m}^{(n-1)}\left(t_{1}\right)=M_{3}\left(\tau-t_{1}\right)^{-\lambda_{1}} \quad \text { and } \quad z_{m}^{(n-1)}\left(\tau_{m}\right) \leq M_{3}\left(\tau-\tau_{m}\right)^{-\lambda_{1}}
$$

or

(ii)

$$
z_{m}^{(n-1)}(t)>M_{3}(\tau-t)^{-\lambda_{1}}
$$

in a left neighborhood of $t=\tau_{m}$.

Let (i) be valid. As $(26)-(30)$ yield $z_{m}^{(n)}(t)>0$ and $z_{m}^{(n-1)}$ is increasing on $J_{m}$, it follows from (32) and (21) that

$$
M \leq z_{m}^{(n-1)}(t), \quad t \in\left[t_{1}, \tau_{m}\right] .
$$

Hence

$$
z_{m}^{(n)}(t) \geq M_{1}\left(z_{m}^{(n-1)}(t)\right)^{\lambda}, \quad t \in J_{m},
$$

and the integration and (32) yield

$$
\frac{\tau_{m}-t_{1}}{M_{3}^{\lambda-1}}>\frac{1}{\left[z_{m}^{(n-1)}\left(t_{1}\right)\right]^{\lambda-1}}-\frac{1}{\left[z_{m}^{(n-1)}\left(\tau_{m}\right)\right]^{\lambda-1}} \geq M_{1}(\lambda-1)\left(\tau_{m}-t_{1}\right),
$$


which contradicts the definition of $M_{3}$.

Let (33) be valid and let $t_{1}, T \leq t_{1}<\tau_{m}$ be such that $z_{m}^{(n-1)}(t)>M_{3}(\tau-t)^{-\lambda_{1}}$ on the interval $\left[t_{1}, \tau_{m}\right)$. Then the Taylor Series Theorem yields

$$
\begin{aligned}
k & =z_{m}^{(l+1)}\left(\tau_{m}\right) \geq \int_{t_{1}}^{\tau_{m}} \frac{\left(\tau_{m}-s\right)^{n-l-3}}{(n-l-3) !} z_{m}^{(n-1)}(s) d s \\
& \geq \frac{M_{3}}{(n-l-3) !} \int_{t_{1}}^{\tau_{m}}\left(\tau_{m}-s\right)^{n-l-3}(\tau-s)^{-\lambda_{1}} d s \\
& \geq \frac{-M_{3}}{(n-l-2) !} \int_{t_{1}}^{\tau_{m}}(\tau-s)^{n-l-1-\lambda_{1}} \frac{d}{d s}\left(\left(1-\frac{1}{m(\tau-s)}\right)^{n-l-2}\right) d s \\
& \geq \frac{M_{3}}{(n-l-2) !}\left(\frac{1}{m}\right)^{n-l-2-\lambda_{1}}\left(1-\frac{1}{m\left(\tau-t_{1}\right)}\right)^{n-l-2} \rightarrow \infty \quad \text { for } m \rightarrow \infty .
\end{aligned}
$$

Hence (31) holds.

Further, we prove indirectly the following estimation from bellow

$$
M<z_{m}^{(n-1)}(t), \quad t \in J_{m} .
$$

Note that $z_{m}^{(n-1)}$ is increasing, and first we prove that (35) is valid for $t=\tau_{m}$. Let, conversely, $z_{m}^{(n-1)}\left(\tau_{m}\right) \leq M$. Then

$k_{0} \leq k=z_{m}^{(l+1)}\left(\tau_{m}\right)=\int_{T}^{\tau_{m}} \frac{\left(\tau_{m}-s\right)^{n-l-3}}{(n-l-3) !} z_{m}^{(n-1)}(s) d s \leq \frac{M}{(n-l-2) !}\left(\tau_{m}-T\right)^{n-l-2}$,

which contradicts (21). Thus (35) holds. Let $T_{1} \in\left[T, \tau_{m}\right)$ exist such that $z_{m}^{(n-1)}\left(T_{1}\right)=M$. Then $M<z_{m}^{(n-1)}(t)$ on $J_{m}$ and

$$
z_{m}^{(n)}(t) \leq M_{2} g\left(z_{m}^{(n-1)}(t)\right), \quad t \in J_{m} .
$$

From this, by the integration, we have

$$
\int_{M}^{2 M} \frac{d s}{g(s)} \leq \int_{M}^{k} \frac{d s}{g(s)} \leq M_{2}\left(\tau_{m}-T\right)<M_{2}(\tau-T) .
$$

The contradiction with (22) proves that (35) is valid and according to (30)

$$
z_{m}^{(j)}(t) \geq 0 \quad \text { on } \quad J_{m}, \quad j=l+1, l+2, \ldots, n .
$$

Denote $\rho=\max _{t \in J_{m}}\left|z_{m}^{(l)}(t)\right|$. Then, by virtue of (31), (36) and Lemma 2 with $[a, b]=[\tau, t], u=z_{m}^{(l)}$ and $m=n-l-1$, we have

$0 \leq z_{m}^{(i+1)}(t) \leq 2^{n-l-2} \rho^{\frac{n-l-2}{n-l-1}}\left(z_{m}^{n-1}(t)\right)^{\frac{1}{n-l-1}} \leq 2^{n-l-2} M_{3}^{\frac{1}{n-l-1}} \rho^{\frac{n-l-2}{n-l-1}}(\tau-t)^{-\frac{\lambda_{1}}{n-l-1}}$, and hence, as $\frac{\lambda_{1}}{n-l-1} \in(0,1)$, the integration on $J_{m}$ yields

$$
0 \leq c_{l}-z_{m}^{(l)}(T) \leq N_{1}(\tau-T)^{1-\frac{\lambda_{1}}{n-l-1}} \rho^{\frac{n-l-2}{n-l-1}} .
$$


Since $z_{m}^{(l)}$ is increasing on $J_{m}$, either $z_{m}^{(l)}(T) \geq-\left|c_{l}\right|$ and $\rho=\left|c_{l}\right|$ or $z_{m}^{(l)}(T)<-\left|c_{l}\right|$ and (23) and (37) yield

$$
c_{l}+\rho \leq N_{1}(\tau-T)^{1-\frac{\lambda_{1}}{n-l-1}} \rho^{\frac{n-l-2}{n-l-1}} \leq \frac{1}{2} N^{\frac{1}{n-l-1}} \rho^{\frac{n-l-2}{n-l-1}} .
$$

Thus $\rho \leq 2\left|c_{l}\right|$ or $\frac{\rho}{2} \leq c_{l}+\rho \leq \frac{1}{2} N^{\frac{1}{n-l-1}} \rho^{\frac{n-l-2}{n-l-1}}$ and according to (20) in all cases we have

$$
\left|z_{m}^{(l)}(t)\right| \leq N, \quad t \in J_{m}
$$

From this, (31), (36) and Lemma 2 with $[a, b]=[T, t], u=z_{m}^{(l)}$ and $m=n-l-1$ we have

$$
\begin{gathered}
\left|z_{m}^{(j)}(t)\right| \leq 2^{(j-l)(n-j-1)} N^{\frac{n-j-1}{n-l-1}} M_{3}^{\frac{j-l}{n-l-1}}(\tau-t)^{\frac{j-l}{n-l-1}}, \\
t \in J_{m}, \quad j=l+1, \ldots, n-2 .
\end{gathered}
$$

Further, (20), (24), (25), (38) and the Taylor Series Theorem yield

$$
\begin{gathered}
c_{i}-z_{m}^{(i)}(t)=\sum_{r=i+1}^{l-1} \frac{c_{r}\left(t-\tau_{m}\right)^{r-i}}{(r-i) !}+\int_{\tau_{m}}^{t} \frac{(t-s)^{l-i-1}}{(l-i-1) !} z_{m}^{(l)}(s) d s, \\
\left|z_{m}^{(i)}(t)\right| \leq \sum_{r=i+1}^{l-1} \frac{c_{r} \mid}{(r-i) !}(\tau-T)^{r-i}+\frac{N}{(l-i) !}(\tau-T)^{l-i}+\left|c_{i}\right| \leq N, \\
i=0,1, \ldots, l-1, \quad t \in J_{m}, \\
\left|z_{m}(t)\right| \geq c_{0}-\sum_{r=1}^{l-1}\left|c_{r}\right| \frac{(\tau-T)^{r}}{r !}-\frac{N}{l !}(\tau-T)^{l} \geq \frac{c_{0}}{2}, \quad t \in J_{m} .
\end{gathered}
$$

Estimations (38), (39) and (40) show that $\left\{z_{m}^{(j)}\right\}, j=0,1, \ldots, n-1, m=$ $m_{0}, m_{0}+1, \ldots$, are uniformly bounded with respect to $j$ and $m$ and hence according to the Arzelá-Ascoli Theorem (see [6], Lemma 10.2) there exists a subsequence that converges uniformly to the solution $y_{k}$ of (26). At the same time, it is clear that (see (41), too)

$$
\begin{gathered}
\frac{c_{0}}{2} \leq y_{k}(t) \leq N,\left|y_{k}^{(i)}(t)\right| \leq N, \quad i=1,2, \ldots, l, \\
\left|y_{k}^{(j)}(t)\right| \leq 2^{(j-l)(n-j-1)} N^{\frac{n-j-1}{n-l-1}} M_{3}^{\frac{j-l}{n-l-1}}(\tau-t)^{\frac{j-l}{n-l-1}}, \\
t \in J, \quad j=l+1, \ldots, n-1 .
\end{gathered}
$$

Moreover, (31), (35), (42) yield

$$
\begin{gathered}
\Phi_{0}\left(y_{k}(t)\right)=y_{k}(t), \quad \Phi_{1}\left(y_{k}^{(i)}(t)\right)=y_{k}^{(i)}(t) \quad \text { for } \quad i=1,2, \ldots, l \\
\Phi_{2}\left(t, y_{k}^{(n-1)}(t)\right)=y_{k}^{(n-1)}(t), \quad t \in J
\end{gathered}
$$

and hence $y_{k}(t)$ is a solution of (1) satisfying

$$
y_{k}^{(i)}(\tau)=c_{i}, \quad i=0,1, \ldots, l ; \quad y_{k}^{(l+1)}(\tau)=k .
$$


As estimations (42) a (43) do not depend on $k, i$ and $j$, the Arzelá-Ascoli Theorem implies the existence of a subsequence of $\left\{y_{k}(\tau)\right\}_{k_{0}}^{\infty}$ that converges uniformly to the solution of (1) satisfying

$$
\begin{gathered}
y^{(j)}(T)=0, \quad j=l+1, \ldots, n-2 \\
\lim _{t \rightarrow \tau_{-}} y^{(i)}(t)=c_{i}, \quad i=0,1, \ldots, l, \quad \lim _{t \rightarrow \tau_{-}} y^{(l)}(t)=\infty .
\end{gathered}
$$

Let $\lambda=1+\frac{1}{n-l-2}$. Then there exists a sequence of $\left\{\lambda_{s}\right\}_{1}^{\infty}$ such that $\lambda_{s}$ satisfies (7) and $\lim _{s \rightarrow \infty} \lambda_{s}=1+\frac{1}{n-l-2}$. Denote by $y_{s}$ a solution of (1), (5) with $\lambda=\lambda_{s}$. It follows from (21)-(25) that there exists $T \in[0, \tau)$ such that $y_{s}, s \in\{1,2, \ldots\}$ is defined on the interval $[T, \tau)$. At the same time, since (38)-(41) do not depend on $\lambda$, there exists $\Phi$ such that

$$
\mid y_{s}^{(i)}(t) \leq \Phi(t), \quad t \in[T, \tau), \quad i=0,1, \ldots, n-1, \quad s=1,2, \ldots .
$$

Hence, according to the Arzelá-Ascoli Theorem, there exists a subsequence of $\left\{y_{s}\right\}_{1}^{\infty}$ that converges uniformly to a solution of (1), satisfying (5).

Let (9) and (10) be valid. Let $y$ be defined on the interval $(\bar{\tau}, \tau) \subset[0, \tau)$ and not be extendable to $t=\bar{\tau}$. Then

$$
\lim \sup _{t \rightarrow \bar{\tau}^{+}}\left|y^{(n-1)}(t)\right|=\infty
$$

First we prove that

$$
y^{(n-1)}(t)>0 \quad \text { on } \quad(\bar{\tau}, \tau) .
$$

Suppose that there exists $\tau_{1} \in(\bar{\tau}, \tau)$ such that $y^{(n-1)}\left(\tau_{1}\right)=0$ and $y^{(n-1)}(t)>0$ on the interval $\left(\tau_{1}, \tau\right)$. As $\tau_{1}<T$, it follows from this and (45) that $y^{(j)}$, $j=0,1, \ldots, l$, are bounded on the interval $\left(\tau_{1}, \tau\right)$. Let $\tau_{2} \in\left(\tau_{1}, \tau\right)$ be such that $y^{(n-1)}\left(\tau_{2}\right)=\varepsilon$. Then by the integration of (1) and (10)

$$
\infty=\int_{0}^{\varepsilon} \frac{d s}{g(s)}=\int_{\tau_{1}}^{\tau_{2}} f\left(t, y(t), \ldots, y^{(l)}(t)\right) d t<\infty .
$$

Hence (47) holds. As $\tau_{1}<T$, it follows from (9), (44) and (45) that $y(t)>0$ on the interval $(\bar{\tau}, \tau)\left(y^{(i)}, i=0,1, \ldots, l\right.$ change their signs). Thus (1) yields $y^{(n)}(t)>0$ on the interval $(\bar{\tau}, \tau)$, which, together with $(47)$, contradicts $(46)$. Hence $y$ is defined at $t=\bar{\tau}$ and $\bar{\tau}=0$.

Proof of Theorem 3. Let $\alpha=1$ and $\beta=1$. The proof is similar to the that of Theorem 2. Since (11) and (12) are valid, we can restrict our investigation to the case

$$
D=\left\{\left[x_{1}, \ldots, x_{l+1}\right] 0 \leq x_{1} \leq \varepsilon,(-1)^{j} x_{j+1} \in\left[(-1)^{j} c_{j},(-1)^{j} c_{j}+\varepsilon\right]\right\} .
$$

The only problem is that due to $c_{0}=0$, we have $M_{1}=0$ and $M_{3}=\infty$, where $M_{1}$ and $M_{3}$ are given as in the proof of Theorem 2. Thus (31) gives us no 
information and it must be proved in a different way. Hence we prove that (31) is valid with the new values of $\lambda_{1}$ and $M_{3}$ given by

$$
\lambda_{1}=\frac{(n-1) \sigma+1}{\lambda+\sigma-1}, \quad M_{3}=\left[\frac{2(n-1) \sigma+2}{(\lambda+\sigma-1) M_{1}}\right]^{\frac{1}{\lambda+\sigma-1}}
$$

where $M_{1}=\frac{\bar{M}}{[l !(n-l-2) !(n-1)]^{\sigma}}$. Note that, similarly to the proof of Theorem 2, $z_{m}^{(n-1)}$ is positively increasing on the interval $J_{m}=\left[T, \tau_{m}\right], \tau_{m}=\tau-\frac{1}{m}$. Note that (13) yields

$$
n-l-2 \leq \lambda_{1}<n-l-1 .
$$

If (31) is not valid, then either (32) or (33) holds.

Let (32) be valid. It follows similarly to (34) that

$$
M \leq z_{m}^{(n-1)}(t), \quad t \in\left[t_{1}, \tau_{m}\right] .
$$

Now we will estimate $z_{m}$. According to (48) and the Taylor Series Theorem we have

$$
\begin{aligned}
z_{m}^{(l+1)}(s) & =\sum_{r=0}^{n-l-3} \frac{z_{m}^{(l+1+r)}(t)}{r !}(s-t)^{r}+\int_{t}^{s} \frac{(s-\sigma)^{n-l-3}}{(n-l-3) !} z_{m}^{(n-1)}(\sigma) d \sigma \\
& \geq z_{m}^{(n-1)}(t) \frac{(s-t)^{n-l-2}}{(n-l-2) !}, \quad t \leq s \leq \tau_{m} .
\end{aligned}
$$

Similarly, the Taylor Series Theorem, (11), (12) and (49) yield

$$
\begin{aligned}
z_{m}(t) & \geq \int_{\tau_{m}}^{t} \frac{(t-s)^{l}}{l !} z_{m}^{(l+1)}(s) d s \geq z_{m}^{(n-1)}(t) \int_{\tau_{m}}^{t} \frac{(s-t)^{n-2}(-1)^{l}}{l !(n-l-2) !} d s \\
& =\frac{\left(\tau_{m}-t\right)^{n-1}}{l !(n-l-2) !(n-1)} z_{m}^{(n-1)}(t), \quad t \in\left[t_{1}, \tau_{m}\right] .
\end{aligned}
$$

From this, (8), (14), (48) and (49)

$$
z_{m}^{(n)}(t) \geq \bar{M} z_{m}^{\sigma}(t)\left(z_{m}^{(n-1)}(t)\right)^{\lambda} \geq M_{1}\left(\tau_{m}-t\right)^{(n-1) \sigma}\left(z_{m}^{(n-1)}(t)\right)^{\lambda+\sigma} .
$$

The integration on the interval $\left[t_{1}, \tau_{m}\right]$ yields

$$
\begin{gathered}
\frac{2\left(\tau_{m}-t_{1}\right)^{(n-1) \sigma+1}}{M_{3}^{\lambda+\sigma-1}} \geq \frac{\left(\tau-t_{1}\right)^{(n-1) \sigma+1}-m^{-(n-1) \sigma+1}}{M_{3}^{\lambda+\sigma-1}} \geq \\
\geq \frac{1}{\left[z_{m}^{(n-1)}\left(t_{1}\right)\right]^{\lambda+\sigma-1}}-\frac{1}{\left[z_{m}^{(n-1)}\left(\tau_{m}\right)\right]^{\lambda+\sigma-1}} \geq \frac{M_{1}(\lambda+\sigma-1)}{(n-1) \sigma+1}\left(\tau_{m}-t_{1}\right)^{(n-1) \sigma+1}
\end{gathered}
$$

for large $m$. The contradiction obtained with the definition of $M_{3}$, shows that (32) does not hold. The fact that (33) is impossible can be proved similarly to the same case in the proof of Theorem 2. 
Proof of Theorem 4. (i) Let $y[T, \tau) \rightarrow \mathbf{R}$ be a solution of (1), (5) with $\alpha=1$ and, for simplicity, $y^{(n-1)}(t) \geq M$ on the interval $[T, \tau)$. Put $\lambda_{1}=\frac{(n-1) \sigma+1}{\lambda+\sigma-1}$ and $M_{1}$ as in the proof of Theorem 3 .

Let $\lambda \geq 1+\frac{(l+1) \sigma+1}{n-l-2}$ for $l<n-2$; hence $n-l-2-\lambda_{1} \geq 0$. We can prove similarly to (44) - (46) that

$$
y^{(n)}(t) \geq M_{1}(\tau-t)^{(n-1) \sigma}\left[y^{(n-1)}(t)\right]^{\lambda+\sigma}, \quad t \in[T, \tau) .
$$

From this and by the integration we obtain an estimation from above of $y^{(n-1)}$ similar to (19) and the proof is similar to the second part of the proof of Theorem 1.

Let $\lambda<1+\frac{l \sigma+1}{n-l-1}$; hence $n-l-1-\lambda_{1}<0$. Then

$$
y(t)=\int_{\tau}^{t} \frac{(t-s)^{l}}{l !} y^{(l+1)}(s) d s \leq \frac{\left|y^{(l)}(t)\right|}{l !}(\tau-t)^{l}, \quad t \in[T, \tau) .
$$

From this

$$
y^{(n)}(t) \leq M_{2} y^{\sigma}(t)\left[y^{(n-1)}(t)\right]^{\lambda} \leq M_{2}(\tau-t)^{l \sigma}\left[y^{(n-1)}(t)\right]^{\lambda},
$$

and the integration on the interval $[t, \tau)$ yields

$$
y^{(n-1)}(t) \geq M_{3}(\tau-t)^{-\frac{l \sigma+1}{\lambda-1}},
$$

where

$$
M_{2}=\max _{t \in[0, \tau]} r(t), \quad M_{3}=\left[\frac{M_{2}(\lambda-1)}{l \sigma+1}\right]^{-\frac{1}{\lambda-1}} .
$$

The proof is similar to the first part of the proof of Theorem 1, only in (18) we take $\lambda_{1}=\frac{l \sigma+1}{\lambda-1}$.

(ii) The existence problem is solved by Theorem 3 .

Proof of Proposition. Let $y[T, \tau) \rightarrow \mathbf{R}$ be a solution of (1) and (5) with $\alpha=1$; hence $l$ is even. Let $\lim _{t \rightarrow \tau^{-}} y^{(n-1)}(t)=\infty$. Then $y^{(l)}<0$ in a left neighborhood $I$ of $\tau$. From this and from $l$ being even we can conclude that $y<0$ and $y^{(n)} \leq 0$ on $I$. The contradiction to $\lim _{t \rightarrow \tau^{-}} y^{(n-1)}=\infty$ proves the statement. Other possible cases can be proved similarly.

\section{ACKNOWLEDGEMENT}

The research of the second author was supported by Grant 201/99/0295 of the Grant Agency of the Czech Republic. 


\section{REFERENCES}

1. M. BartušEK, On Existence of singular solutions of $n$-th order differential equations. Arch. Math. (Brno) 36(2000), 395-404.

2. T. A. Chanturia, On existence of singular and unbounded oscillatory solutions of differential equations of Emden-Fowler type. Diffrentsial'nye Uraveniya 28(1992), 1009-1022.

3. J. JAROŠ and T. KusANo, On black hole solutions of second order differential equations with a singularity in the differential operator. Funk. Ekv. (to appear).

4. I. T. Kiguradze, Asymptotic properties of solutions of a nonlinear differential equation of Emden-Fowler type. Izv. Akad. Nauk SSSR, Ser. Mat. 29(1965), 965-986.

5. I. T. Kiguradze, Some singular boundary-value problems for ordinary differential equations. (Ruissian) Tbilisi University Press, Tbilisi, 1975.

6. I. T. Kiguradze and T. Chanturia, Asymptotic properties of solution of nonautonomous ordinary differential equations. Kluwer Academic Publishers, Dordrecht, 1993.

(Received 10.04.2001)

Authors' address:

Department of Mathematics

Masaryk University

Janáčkovo nám. 2a, 66295 Brno

Czech Republic

E-mail: Bartusek@math.muni.cz

Osicka@math.muni.cz 